\title{
ON THE CHAOS GAME OF ITERATED FUNCTION SYSTEMS
}

\author{
PABLO G. BARRIENTOS — FATEMEH H. GHANE \\ DOMINIQUE MALICET — ALIASGHAR SARIZADEH
}

\begin{abstract}
Aвstract. Every quasi-attractor of an iterated function system (IFS) of continuous functions on a first-countable Hausdorff topological space is renderable by the probabilistic chaos game. By contrast, we prove that the backward minimality is a necessary condition to get the deterministic chaos game. As a consequence, we obtain that an IFS of homeomorphisms of the circle is renderable by the deterministic chaos game if and only if it is forward and backward minimal. This result provides examples of attractors (a forward but no backward minimal IFS on the circle) that are not renderable by the deterministic chaos game. We also prove that every well-fibred quasi-attractor is renderable by the deterministic chaos game as well as quasi-attractors of both, symmetric and non-expansive IFSs.
\end{abstract}

\section{INTRODUCTION}

Within fractal geometry, iterated function systems (IFSs) provide a method for both generating and characterizing fractal images. An iterated function system (IFS) can also be thought of as a finite collection of functions which can be applied successively in any order. Attractors of this kind of systems are self-similar compact sets which draw any iteration of any point in an open neighborhood of itself.

There are two methods for generating the attractor: the deterministic algorithm, in which all the transformations are applied simultaneously, and the random algorithm, in which the transformations are applied one at a time in random order following a probability. The chaos game, popularized by Barnsley [3], is the simple algorithm implementing the random method. We have two different forms to run the chaos game. One involves taking a starting point and then choose randomly the transformation on each iteration accordingly to the assigned probabilities. The latter starts by choosing a random order iteration and then applying this orbital branch anywhere in the basin of attraction. The first form of implementation is called probabilistic chaos game $[9,7]$. The second implementation is called deterministic chaos game (also called disjunctive chaos game) [27, 5, 12].

2010 Mathematics Subject Classification. Primary: 37C05, 37C20; Secondary: 37E10

Key words and phrases. iterated function system, well-fibred attractors, deterministic and probabilistic chaos game, forward and backward minimality.

The first author was partially supported by Ministerio de Ciencia e Innovación project MTM2014-56953-P. 
According to [7], every attractor of an IFS of continuous maps on a first- countable Hausdorff topological space is renderable by the probabilistic chaos game. By contract, we will see that this is not the case of the deterministic chaos game. Namely, we will provide necessary and sufficient conditions to get the deterministic chaos game. As an application we will obtain that an IFS of homeomorphisms of the circle is renderable by the deterministic chaos game if and only if it is forward and backward minimal which provides examples of attractors that are not renderable by the deterministic chaos game.

1.1. Iterated function systems. Let $X$ be a Hausdorff topological space. We consider a finite set $\mathscr{F}=\left\{f_{1}, \ldots f_{k}\right\}$ of continuous functions from $X$ to itself. Associated with this set $\mathscr{F}$ we define the semigroup $\Gamma=\Gamma_{\mathscr{F}}$ generated by these functions, the Hutchinson operator $F=F_{\mathscr{F}}$ on the hyperspace $\mathscr{H}(X)$ of the non-empty compact subsets of $X$

$$
F: \mathscr{H}(X) \rightarrow \mathscr{H}(X), \quad F(A)=\bigcup_{i=1}^{k} f_{i}(A)
$$

and the skew-product $\Phi=\Phi_{\mathscr{F}}$ on the product space of $\Omega=\{1, \ldots, k\}^{\mathbb{N}}$ and $X$

$$
\Phi: \Omega \times X \rightarrow \Omega \times X, \quad \Phi(\omega, x)=\left(\sigma(\omega), f_{\omega_{1}}(x)\right),
$$

where $\omega=\omega_{1} \omega_{2} \cdots \in \Omega$ and $\sigma: \Omega \rightarrow \Omega$ is the lateral shift map. The action of the semigroup $\Gamma$ on $X$ is called the iterated function system generated by $f_{1}, \ldots, f_{k}$ (or, by the family $\mathscr{F}$ for short). Finally, given $\omega=\omega_{1} \omega_{2} \cdots \in \Omega$ and $x \in X$,

$$
O_{\omega}^{+}(x)=\left\{f_{\omega}^{n}(x): n \in \mathbb{N}\right\} \quad \text { where } f_{\omega}^{n} \stackrel{\text { def }}{=} f_{\omega_{n}} \circ \cdots \circ f_{\omega_{1}} \quad \text { for every } n \in \mathbb{N},
$$

are called, respectively, the $\omega$-fiberwise orbit of $x$ and the orbital branch corresponding to $\omega$ (or the IFS-iteration driving by the sequence $\omega$ ). We introduce now some different notions of invariant and minimal sets and after that we give the definition of an attractor. In what follows $A$ denotes a closed subset of $X$.

1.2. Invariant and minimal sets. We say that $A$ is a forward invariant set if $f(A) \subset A$ for all $f \in \Gamma$. We also say that $A$ is a self-similar set if

$$
A=f_{1}(A) \cup \cdots \cup f_{k}(A) .
$$

Notice that a minimal set regarding to the inclusion of forward invariant non-empty (closed) sets is always a self-similar set. We simply call it as forward invariant minimal set. By extension, we say that the IFS is forward minimal if the unique forward invariant non-empty closed set is the whole space. It is not difficult to see that forward minimality is equivalent to density of any $\Gamma$-orbit. That is, $A$ is a forward invariant minimal set if and only if $A$ coincides with the closure of $\Gamma$-orbit $\Gamma(x) \stackrel{\text { def }}{=}\{g(x): g \in \Gamma\}$ for all $x \in A$. Similarly, we will say that $A$ is a forward minimal set if $A$ is contained in the closure of $\Gamma(x)$ for all $x \in A$. Thus, forward minimal self-similar sets are forward invariant minimal sets and viceversa.

Definition 1.1. We say that $A$ is a quasi-attractor of the IFS generated by $\mathscr{F}$ if it is a forward minimal self-similar compact set, i.e., if $A \in \mathscr{H}(X), F(A)=A$ and $A=\overline{\Gamma(x)}$ for all $x \in A$. 
Finally, notice that, as a straightforward application of Zorn's lemma, every IFS on a compact space has a quasi-attractor.

1.3. Attractors. We introduce the notion of attractor following [9, 10, 8, 7]. To accomplish this, we need to define first the pointwise basin of attraction.

Given a compact set $K$ of $X$, the Ls-limit set (also called $\omega$-limit set or topological upper limit set) of $K$ for $F$ is the set

$$
\operatorname{Ls} F^{n}(K) \stackrel{\text { def }}{=} \bigcap_{m \in \mathbb{N}} \overline{\bigcup_{n \geq m} F^{n}(K)} .
$$

Observe that $\operatorname{Ls} F^{n}(S)$ is always closed. However it can be non-compact. Now, let $A$ be a compact set. The pointwise basin of Ls-attraction of $A$ for $F$ is defined to be the set

$$
\mathcal{B}_{p}^{*}(A) \stackrel{\text { def }}{=}\left\{x \in X: \operatorname{Ls}^{n}(\{x\})=A\right\} .
$$

Similarly, the pointwise basin of Vietoris-attraction for $F$ is the set

$$
\mathcal{B}_{p}(A) \stackrel{\text { def }}{=}\left\{x \in X: \lim _{n \rightarrow \infty} F^{n}(\{x\})=A\right\} .
$$

The convergence here is with respect to the Vietoris topology, or equivalently, in the metric space case, with respect to the Hausdorff metric [31, pp. 66-69]. Moreover, it is not difficult to show that $\mathcal{B}_{p}(A) \subset \mathcal{B}_{p}^{*}(A)$.

A compact set $A$ is a pointwise attractor if there is an open set $U$ of $X$ such that $A \subset U \subset \mathcal{B}_{p}(A)$. A slightly stronger notion of an attractor is the following. $A$ is said to be a strict attractor if there is an open neighborhood $U$ of $A$ such that $\lim _{n \rightarrow \infty} F^{n}(K)=A$ in the Vietoris topology for all compact sets $K \subset U$. We denote by $\mathcal{B}(A)$ the basin of the strict attractor. That is, the union of all open neighborhoods $U$ of $A$ such that the above convergence holds.

We remark that it is usual to include in the definition of attractor that $F(A)=A$ (cf. [10, Def. 2.2]). Under our mild assumptions on $X$, it is unknown the continuity of the Hutchinson operator (see [6]) and thus it is not, a priori, clear that $A$ is a self-similar (F-invariant) set for the IFS. Nevertheless, the following result proves that any attractor must be a quasi-attractor and, in the case of a strict attractor, must attract any compact set in the basin of attraction which, a priori, is also not clear from the definition.

Theorem A. Consider the IFS generated by $\mathscr{F}$ and a compact subset $A$.

(1) $A$ is a quasi-attractor if and only if $A \subset \mathcal{B}_{p}^{*}(A)$. Moreover, in this case,

$$
A=\operatorname{Ls} F^{n}(K) \text { for all non-empty compact sets } K \subset A \text {; }
$$

(2) If $A$ is a pointwise attractor, it is a quasi-attractor and $\mathcal{B}_{p}(A)=\mathcal{B}_{p}^{*}(A)$;

(3) If $A$ is a strict attractor, it is a pointwise attractor, $\mathcal{B}(A)=\mathcal{B}_{p}(A)$ and for every non-empty compact set $K \subset \mathcal{B}(A)$

$$
\lim _{n \rightarrow \infty} F^{n}(K)=A \text { in the Vietoris topology. }
$$


Another notion of the "attractor" of an IFS is the concept of semi-attractor introduced by Lasota and Myjak in [24]. Semi-attractors, sometimes called semi-fractals, are the smallest (unique) forward invariant set defined by means of the Kuratowski topological limits. We refer to $[25,26]$ for a precise definition. Thus, these sets are also forward invariant selfsimilar sets (in particular closed sets) but in contracts with strict/pointwise attractors or quasi-attractors, semi-attractors can be non-compact.

Examples of pointwise attractors that are not strict attractors can be find in [7]. Also, one easily can construct quasi-attractors of IFSs that are neither attractors nor semi-attractor. A simple example is provided by an IFS generated by a minimal map $f$ (for instance a rotation of the circle with irrational rotation number). The whole space $A$ is the unique non-empty forward invariant closed set but it is not the limit in the Hausdorff metric of $F^{n}(\{x\})=\left\{f^{n}(x)\right\}$ for any $x \in A$. However, it always holds that $\mathcal{B}_{p}^{*}(A)=A$.

1.4. Chaos game. Now, we focus our study to the chaos game of quasi-attractors of the IFS generated by $\mathscr{F}$ on $X$. In particular, this covers the cases of pointwise attractors, strict attractors, compact semi-attractors and minimal IFSs on a compact topological space. First, we will give a rigourously definition of the chaos game.

Following [9], we consider any probability $\mathbb{P}$ on $\Omega$ with the following property: there exists $0<p \leq 1 / k$ so that $\omega_{n}$ is selected randomly from $\{1, \ldots, k\}$ in such a way that the probability of $\omega_{n}=i$ is greater than or equal to $p$, regardless of the preceding outcomes, for all $i \in\{1, \ldots, k\}$ and $n \in \mathbb{N}$. More formally, in terms of the conditional probability,

$$
\mathbb{P}\left(\omega_{n}=i \mid \omega_{n-1}, \ldots, \omega_{1}\right) \geq p .
$$

Bernoulli measures on $\Omega$ are typical examples of these kinds of probabilities.

Definition 1.2. Let $A$ be a quasi-attractor of the IFS generated by $\mathscr{F}$. We say that $A$ is renderable by

(1) the probabilistic chaos game if for any $x \in \mathcal{B}_{p}^{*}(A)$ there is $\Omega(x) \subset \Omega$ with $\mathbb{P}(\Omega(x))=1$ such that

$$
A \subset \overline{O_{\omega}^{+}(x)} \text { for all } \omega \in \Omega(x) \text {; }
$$

(2) the deterministic chaos game if there is $\Omega_{0} \subset \Omega$ with $\mathbb{P}\left(\Omega_{0}\right)=1$ such that

$$
A \subset \overline{O_{\omega}^{+}(x)} \text { for all } \omega \in \Omega_{0} \text { and } x \in \mathcal{B}_{p}^{*}(A) \text {. }
$$

If the IFS is forward minimal (consequently $A$ is the whole space and $\mathcal{B}_{p}^{*}(A)=A$ ) we simply say that the IFS is renderable by the probabilistic/deterministic chaos game.

The sequences in $\Omega$ which have a dense orbit under the shift map $\sigma: \Omega \rightarrow \Omega$ are called disjunctive. That is, the sequence $\omega=\omega_{1} \omega_{2} \cdots \in \Omega$ which contains all the finite words $\alpha=\alpha_{1} \ldots \alpha_{n} \in\{1, \ldots, k\}^{n}$ of length $n$, for all $n \geq 1$. Notice that the set consisting of all disjunctive sequences has $\mathbb{P}$-probability one and its complement is a $\sigma$-porous set with respect to the Baire metric in $\Omega$ [5]. The following result shows that the existence of a 
sequence $\omega$ such that every point in the basin of attraction has dense $\omega$-fiberwise orbit on the self-similar set is enough to guarantee that for any disjunctive sequence we can also draw the quasi-attractor. This brings to light that actually the deterministic chaos game does not depend on the probability $\mathbb{P}$. This fact deradomizes the algorithm of the chaos game since disjunctive sequences in $\Omega$ are a priori well determined sequences. For this reason, the algorithm is called the deterministic chaos game (or disjunctive chaos game).

Theorem B. Consider the IFS generated by $\mathscr{F}$ and let $A$ be a compact set of $X$ and $x \in \mathcal{B}_{p}^{*}(A)$. Then,

(1) $A$ and $\overline{\Gamma(x)}$ are forward invariant compact sets of $X$ and $A \subset \overline{\Gamma(x)}$. In particular, for every $n \in \mathbb{N}$ and $\omega \in \Omega$,

$$
\overline{\left\{f_{\omega}^{m}(x): m \geq n\right\}} \text { is a compact set; }
$$

(2) $A \subset \overline{O_{\omega}^{+}(x)}$ if and only if

$$
\lim _{n \rightarrow \infty} \overline{\left\{f_{\omega}^{m}(x): m \geq n\right\}}=A \text { in the Vietoris topology; }
$$

(3) if $A$ is a quasi-attractor, the following are equivalent:

(a) A renderable by the deterministic chaos game;

(b) there is $\omega \in \Omega$ such that $A \subset \overline{O_{\omega}^{+}(z)}$ for all $z \in \mathcal{B}_{p}^{*}(A)$;

(c) $A \subset \overline{O_{\omega}^{+}(z)}$ for all $z \in \mathcal{B}_{p}^{*}(A)$ and disjunctive sequences $\omega \in \Omega$.

1.4.1. Probabilistic chaos game. Initially, the method was developed for contracting IFSs [3]. Later, it was generalized to attractors of IFSs of continuous functions on proper metric spaces [9]. For minimal IFSs in the case of independent identically distributed random product of continuous maps of a compact metric space it is follows from the Breiman's law of large numbers [17]. Recently in [7], Barnsley, Leśniak and Rypka proved the probabilistic chaos game for pointwise attractors of continuous IFSs on a first-countable Hausdorff topological space (in fact they only need to assume that the attractor is first-countable). Moreover, their proof of the probabilistic chaos game also works for the general case of quasi-attractors with minor modifications (see Appendix A).

1.4.2. Deterministic chaos game. In the case of attractors of contractive IFSs a very simple justification of the deterministic chaos game can be given along the lines in [18, proof of Thm 5.1.3]. In [11], the deterministic algorithm was also proved for attractors of weakly hyperbolic IFSs, i.e., for point-fibred attractors (see the below definition), which are an extension of the previous attractors of contractive IFSs. Later, in [5] the deterministic chaos was obtained for a more general class of attractors, the so-called strongly-fibred.

An attractor $A$ is said to be strongly-fibred if for every open set $U \subset X$ such that $U \cap A \neq \emptyset$, there exists $\omega \in \Omega$ so that

$$
A_{\omega} \stackrel{\text { def }}{=} \bigcap_{n=1}^{\infty} f_{\omega_{1}} \circ \cdots \circ f_{\omega_{n}}(A) \subset U .
$$

Similarly, $A$ is said to be point-fibred if $A_{\omega}$ is a singleton for all $\omega \in \Omega$. Same definitions will lead to quasi-attractors. We are going to introduce a similar category that we will call well-fibred following the proposal Kieninger's classification of IFS attractors [22, p. 97], [8]. 
Definition 1.3. We say that a quasi-attractor $A$ of the IFS is well-fibred if for every compact set $K$ in $A$ so that $K \neq A$ and for any open cover $\mathcal{U}$ of $A$, there exist $g \in \Gamma$ and $U \in \mathcal{U}$ such that $g(K) \subset U$. Equivalently, if there are $\omega \in \Omega$ and $U \in \mathcal{U}$ so that

$$
K_{\omega} \stackrel{\text { def }}{=} \bigcap_{n=1}^{\infty} f_{\omega_{1}} \circ \cdots \circ f_{\omega_{n}}(K) \subset U .
$$

It is not difficult to see that, in the metric space case, a quasi-attractor $A$ is well-fibred if and only if for every compact set $K$ in $A$ so that $K \neq A$, there is a sequence $\left(g_{n}\right)_{n} \subset \Gamma$ such that the diameter diam $g_{n}(K)$ converges to zero as $n \rightarrow \infty$. On the other hand, it is easy to show that strongly-fibred implies well-fibred. In fact, we will prove that if $f_{i}(A)$ is not equal to $A$ for some generator $f_{i}$ then both notions, strongly-fibred and well-fibred, are equivalent. After this observation, we can say that the following result generalizes [5].

Theorem C. Every well-fibred quasi-attractor A of an IFS of continuous maps of a Hausdorff topological space is renderable by the deterministic chaos game. Moreover, if A is either, stronglyfibred or the generators of the IFS restricted to A are homeomorphisms, then

$$
\Omega \times A=\overline{\left\{\Phi^{n}(\omega, x): n \in \mathbb{N}\right\}} \text { for all disjunctive } \omega \in \Omega \text { and } x \in A .
$$

As a consequence, we will prove that every forward and backward minimal IFS of homeomorphisms $\mathscr{F}$ of a metric space so that the associated semigroup has a map with exactly two fixed points, one attracting and one repelling, is renderable by the deterministic chaos game (see Corollary 3.17). Backward minimality here means that the IFS generated by $\mathscr{F}^{-1}=\left\{f^{-1}: f \in \mathscr{F}\right\}$ is forward minimal.

New examples of attractors renderable by the deterministic chaos game which are not necessarily well-fibred were given in [12,27]. Namely, in [12] the deterministic chaos was proved for any forward and backward minimal IFS of homeomorphisms of the circle and for every IFS of a compact metric space that contains a minimal map. In [27] the deterministic algorithm was shown to work also for attractors of IFSs comprising maps which do not increase distances. In fact, basically with the same proof (see Appendix A), the result of Leśniak also holds for quasi-attractors of non-expansive IFSs, i.e., iterated function systems generated by a finite family $\mathscr{F}$ of maps of a metric space $X$ so that

$$
d(f(x), f(y)) \leq d(x, y) \quad \text { for all } f \in \mathscr{F} .
$$

This class of systems include equicontinuous IFSs (see [28, Lem. 3.2] and [33, Prop. 8]) and weakly hyperbolic IFSs (see [4, Thm. 1] and [2, Cor. 6.4]). However, a priori, there are no relations between quasi-attractors of non-expansive IFSs and strongly-fibred or well-fibred attractors.

In brief, it is known that the deterministic chaos algorithm holds in the following cases:

(1) well-fibred quasi-attractors of IFSs on Hausdorff topological spaces,

(2) quasi-attractors of non-expansive IFSs on metric spaces,

(3) forward and backward minimal IFSs of homeomorphisms of the circle,

(4) IFSs on a compact metric space having a minimal map. 
The following theorem includes another different class of systems to this list: the quasiattractors of symmetric IFSs. We say that an IFS generated by a family of homeomorphisms $\mathscr{F}$ of $X$ is symmetric if for each $f \in \mathscr{F}$ it holds that $f^{-1} \in \mathscr{F}$.

Theorem D. Every quasi-attractor of a symmetric IFS on a Hausdorff topological space is renderable by the deterministic chaos game.

We will give examples of symmetric non-minimal IFSs with a quasi-attractor which is not an attractor (Remark 3.4) and attractors of symmetric IFSs which are not include in the previous list (Exemple 3.19). Moreover, we will prove that the phase space of a forward minimal symmetric IFS on a connected space is a strict atractor (Proposition 3.7).

1.4.3. Necessary condition to get the deterministic chaos game. The next result goes in the direction to provide necessarily conditions to yield the deterministic chaos game. First we need to introduce the notion of backward minimality. A set $A$ of $X$ is said to be backward invariant for the IFS if $\emptyset \neq f^{-1}(A) \subset A$ for all $f \in \Gamma$, where $f^{-1}(A)$ denotes the preimage of $A$ by the continuous map $f$. Hence, we say that the IFS is backward minimal if the unique backward invariant non-empty closed set is the whole space.

Theorem E. Every forward minimal IFS generated by continuous maps of a compact Hausdorff topological space which is renderable by the deterministic chaos game must be also backward minimal.

As an application of the above result we can complete the main result in [12] obtaining the following corollary:

Corollary I. Let $f_{1}, \ldots, f_{k}$ be circle homeomorphisms. Then the following statements are equivalent:

(1) the IFS generated by $f_{1}, \ldots, f_{k}$ is renderable by the deterministic chaos game;

(2) there exists $\omega \in \Omega$ such that $\overline{O_{\omega}^{+}(x)}=S^{1}$ for all $x \in S^{1}$;

(3) the IFS generated by $f_{1}, \ldots, f_{k}$ is forward and backward minimal.

This result allows us to construct a contra-example of the deterministic chaos game for general IFSs. More specifically, any forward minimal but not backward minimal IFS of homeomorphisms of the circle is not renderable by the deterministic chaos game. Observe that for ordinary dynamical systems on the circle, the minimality of a map $T$ is equivalent to that of $T^{-1}$. However this fact does not hold for IFSs with more than one generator:

Corollary II. There exists an IFS of homeomorphisms of the circle that is forward minimal but not backward minimal. Moreover, $S^{1}$ is a strict attractor of this IFS which, consequently, is not renderable by the deterministic chaos game.

We want to indicate that, as we will see, most of the minimal IFSs of homeomorphisms of the circle have $S^{1}$ as an strict attractor. Namely, we will prove that $S^{1}$ is an strict attractor of a minimal IFS of homeomorphisms of the circle if there is no common invariant measure for the generators (Proposition 3.9). 
Organization of the paper: In $\S 2$ we study the basin of attraction of pointwise/strict attractors and we prove Theorem A and the two first conclusions of Theorem B. We complete the proof of Theorem B in $\$ 3.1$ where we study the deterministic chaos game. In $\$ 3.2$ we prove Theorem E and in $\S 3.3$ we study the deterministic chaos game on the circle proving Corollaries I and II. The proof of Theorems C and D are developed in $\$ 3.4$ where we study sufficient conditions for the deterministic chaos game. Finally, for completeness of the paper, we include an appendix where we extend the main results of [7] and [27] for the general case of quasi-attractors.

Standing notation: In the sequel, $X$ denotes a Hausdorff topological space. We assume that we work with an IFS of continuous maps $f_{1}, \ldots, f_{k}$ on $X$ and we hold the above notations introduced in this section.

\section{ON THE BASIN OF ATtRACTION}

We will study the basin of attraction of quasi-attractors. This allows us to prove Theorem $\mathrm{A}$ and Conclusions (1) and (2) of Theorem B.

2.1. Topological preliminaries: We start giving a basic topological lemma:

Lemma 2.1. Let $A$ and $B$ be two compact sets in $X$.

(1) If $A \cap B=\emptyset$ then there exist disjoint open neighborhoods of $A$ and $B$;

(2) If $\left\{U_{1}, \ldots, U_{s}\right\}$ is a finite open cover of $A$ then there exist compact sets $A_{1}, \ldots, A_{s}$ in $X$ so that

$$
A=A_{1} \cup \cdots \cup A_{s} \text { and } A_{i} \subset U_{i} \text { for } i=1, \ldots, s .
$$

Proof. The first conclusion is a well known equivalent definition of a Hausdorff topological space (see [30, Lemma 26.4 and Exercice 26.5]). Hence, we only need to prove the second conclusion. First of all, notice that it suffices to prove the result for an open cover of $A$ with two sets. So, let $\left\{U_{1}, U_{2}\right\}$ be an open cover of $A$. Since $X$ is Hausdorff, $A$ is a closed subset of $X$. Let us consider compact subsets $K_{1}=A \backslash U_{2} \subset U_{1}$ and $K_{2}=A \backslash U_{1} \subset U_{2}$. If $A \subset K_{1} \cup K_{2}$ then we set $A_{1}=K_{1}, A_{2}=K_{2}$ and it is done. Otherwise, $A \backslash\left(K_{1} \cup K_{2}\right)$ is a nonempty subset of $A$ and it is easy to see that $K_{1} \cap K_{2}=\emptyset$. Since $X$ is Hausdorff and $K_{1}$ and $K_{2}$ are compact disjoint subsets of $X$, by the first conclusion, there are disjoint open subsets $V_{i}$ of $X$ so that $K_{i} \subset V_{i}$, for $i=1,2$. We may assume that $V_{i} \subset U_{i}$. Now let us take $A_{1}=A \backslash V_{2} \subset U_{1}$ and $A_{2}=A \backslash V_{1} \subset U_{2}$. Then $A_{1}$ and $A_{2}$ are compact subsets of $X$ and $A=A_{1} \cup A_{2}$ which concludes the proof.

Let $A$ and $A_{n}, n \geq 1$, be compact subsets of $X$. Following [15], we define the upper Kuratowski limit of $\left(A_{n}\right)_{n}$ as the set

$$
\operatorname{Ls} A_{n} \stackrel{\text { def }}{=} \bigcap_{m \geq 1} \overline{\bigcup_{n \geq m} A_{n}} .
$$


Observe that $\operatorname{Ls} A_{n}$ is a closed set and $\operatorname{Ls} A_{n} \subset B$ that provide $A_{n} \subset B$, for $n$ sufficiently large, and $B$ is a closed set. On the other hand, we recall that the Vietoris topology in $\mathscr{H}(X)$ is generated by the basic sets of the form

$$
O\left\langle U_{1}, \ldots, U_{m}\right\rangle=\left\{K \in \mathscr{H}(X): K \subset \bigcup_{i=1}^{m} U_{i}, K \cap U_{i} \neq \emptyset \text { for } k=1, \ldots, m\right\}
$$

where $U_{1}, \ldots U_{m}$ are open sets in $X$ and $m \in \mathbb{N}$. Hence, if $A_{n} \rightarrow A$ in the Vietoris topology then $A_{n} \in O\langle U\rangle$ for any $n$ large enough and any open set $U$ in $X$ such that $A \subset U$. In particular $A_{n} \subset U$ for all $n$ sufficiently large. Moreover we have the following:

Lemma 2.2. $A_{n} \rightarrow A$ in the Vietoris topology if and only if for any pair of open sets $U$ and $V$ such that $A \subset U$ and $A \cap V \neq \emptyset$, there is $n_{0} \in \mathbb{N}$ so that

$$
\bigcup_{n \geq n_{0}} A_{n} \subset U \text { and } V \cap A_{n} \neq \emptyset \text { for all } n \geq n_{0} .
$$

In particular,

$$
A=\operatorname{Ls} A_{n} \stackrel{\text { def }}{=} \bigcap_{m \geq 1} \overline{\bigcup_{n \geq m} A_{n}}
$$

Proof. Assume that $A_{n} \rightarrow A$ in the Vietoris topology. Let $U$ be any open set such that $A \subset U$. By applying the above observation, there is $n_{0} \in \mathbb{N}$ such that $A_{n} \subset U$ for all $n \geq n_{0}$. Now we will see that for any open set $V$ with $A \cap V \neq \emptyset$, it holds that $A_{n} \cap V \neq \emptyset$ for all $n$ sufficiently large. By the compactness of $A$, we extract open sets $U_{1}, \ldots, U_{s}$ in $X$ such that

$$
A \cap U_{i} \neq \emptyset \text { and } A \subset V \cup U_{1} \cup \cdots \cup U_{s} .
$$

Hence $O\left\langle V, U_{1}, \ldots, U_{s}\right\rangle$ is an open neighborhood of $A$ in $\mathscr{H}(X)$. Since $A_{n}$ converges to $A$ then $A_{n} \in O\left\langle V, U_{1}, \ldots, U_{s}\right\rangle$ for all $n$ large enough and in particular $A_{n} \cap V \neq \emptyset$ for all $n$ large.

We will prove the converse. Let $O\left\langle U_{1}, \ldots, U_{m}\right\rangle$ be an basic open neighborhood of $A$. Thus, $U_{1}, \ldots, U_{m}$ are open sets in $X$ and

$$
A \subset U_{1} \cup \cdots \cup U_{m} \stackrel{\text { def }}{=} U \text { and } A \cap U_{i} \neq \emptyset \text { for all } i=1, \ldots, m .
$$

By assumption, there is $n_{0}$ such that $A_{n} \subset U$ for all $n \geq n_{0}$. Moreover, since $A \cap U_{i} \neq \emptyset$, also we get $n_{i}$ such that $A_{n} \cap U_{i} \neq \emptyset$ for all $n \geq n_{i}$ and $i=1, \ldots, m$. Therefore

$$
A_{n} \in O\left\langle U_{1}, \ldots, U_{m}\right\rangle \text { for all } n \geq N=\max \left\{n_{i}: i=0, \ldots, m\right\} .
$$

This implies that $A_{n} \rightarrow A$ in the Vietoris topology.

Finally we will prove (2). We have that $A \subset \operatorname{Ls} A_{n}$ since for every open neighborhood $V$ of any point in $A$ there is $n_{0} \in \mathbb{N}$ such that

$$
A_{n} \cap V \neq \emptyset \text { for all } n \geq n_{0} .
$$

Reverse content is equivalent to prove that for every compact set $K$ such that $K \cap A=\emptyset$, there exists $n_{0} \in \mathbb{N}$ so that $A_{n} \cap K=\emptyset$ for all $n \geq n_{0}$. But this is a consequence again of Lemma 2.1. Indeed, since $K$ and $A$ are compact sets, we can find disjoint open sets $U$ and $V$ such that 
$A \subset U$ and $K \subset V$. By the above characterization of the Vietoris convergence, there is $n_{0} \in \mathbb{N}$ such that $A_{n} \subset U$ for all $n \geq n_{0}$. In particular $A_{n} \cap K=\emptyset$ for all $n \geq n_{0}$.

2.2. Proof of Theorem A and Conclusions (1)-(2) of Theorem B. We start proving Conclusion (1) of Theorem B.

Proposition 2.3. Let $A$ be a compact subset of $X$. If $x \in \mathcal{B}_{p}^{*}(A)$ then both, $A$ and $\overline{\Gamma(x)}$, are forward invariant compact sets such that

$$
A=\operatorname{Ls} F^{n}(\{x\}) \stackrel{\text { def }}{=} \bigcap_{m \geq 1} \overline{\bigcup_{n \geq m} F^{n}(\{x\})} \text { and } \overline{\Gamma(x)}=\bigcup_{n \geq 1} F^{n}(\{x\}) \cup A .
$$

In particular,

$$
\overline{\left\{f_{\omega}^{m}(x): m \geq n\right\}} \text { is a compact set for all } n \in \mathbb{N} \text { and } \omega \in \Omega \text {. }
$$

Proof. Set $K \stackrel{\text { def }}{=} \overline{\Gamma(x)}$. Since $x \in \mathcal{B}_{p}^{*}(A)$, by definition it follows the above characterization of $A$, and consequently of $K$.

Now we will show that $K$ is compact. Let $\left\{U_{\alpha}: \alpha \in I\right\}$ be an open cover of $K$. Since $A \subset K$, by the compactness of $A$ there exists a finite subset $J_{1}$ of $I$ such that

$$
A \subset \bigcup_{\alpha \in J_{1}} U_{\alpha} \stackrel{\text { def }}{=} U \text {. }
$$

Again, by the above characterization of the set $A$ and since

$$
\bigcup_{n \geq m} F^{n}(\{x\}) \text { for } m \geq 1
$$

is a nested sequence then there is $n_{0} \in \mathbb{N}$ such that the union of $F^{n}(\{x\})$ for $n \geq n_{0}$ is contained in $U$. On the other hand, the set $F(\{x\}) \cup \cdots \cup F^{n_{0}-1}(\{x\})$ is a finite union of compact sets and thus, it is compact. Hence, there is a finite subset $J_{2}$ of $I$ such that

$$
F(\{x\}) \cup \cdots \cup F^{n_{0}-1}(\{x\}) \subset \bigcup_{\alpha \in J_{2}} U_{\alpha} .
$$

Put together all and setting $J=J_{1} \cup J_{2}$ we get that

$$
K=\overline{\Gamma(x)}=A \cup F(\{x\}) \cup \cdots \cup F^{n_{0}-1}(\{x\}) \cup \bigcup_{n \geq n_{0}} F^{n}(\{x\}) \subset \bigcup_{\alpha \in J} U_{\alpha}
$$

concluding that $K$ is compact.

Moreover clearly $F(K) \subset K$. Thus we have obtained that $K$ is a compact Hausdorff topological space so that $F(K) \subset K$ and $A \subset K$. Hence, we can restrict the map $F$ to the set of non-empty compact subsets of $K$.

According to [22, Prop. 1.5.3 (iv)], see also [6], the Hutchinson operator $F: \mathscr{K}(K) \rightarrow \mathscr{K}(K)$ is continuous and from the above characterization of the set $A$, it is easy to conclude that $A$ is also a forward invariant compact set. This completes the proof of the proposition.

Now, we characterize the quasi-attractors (Conclusions (1)-(2) of Theorem A). 
Proposition 2.4. Let $A$ be a compact subset of X. Then

(1) $\mathcal{B}_{p}(A) \subset \mathcal{B}_{p}^{*}(A)$;

(2) $A$ is a quasi-attractor if and only if $A \subset \mathcal{B}_{p}^{*}(A)$. Moreover, in this case,

Ls $F^{n}(K)=A \quad$ for all non-empty compact set $K \subset A$ and

$$
\overline{\Gamma(x)} \subset \mathcal{B}_{p}^{*}(A) \text { for all } x \in \mathcal{B}_{p}^{*}(A) \text {; }
$$

(3) if $A$ is a pointwise attractor, it is a quasi-attractor and $\mathcal{B}_{p}(A)=\mathcal{B}_{p}^{*}(A)$.

Proof. The first conclusion follows from the characterization (2) of the limit of $F^{n}(\{x\})$ in the Vietoris topology given in Lemma 2.2.

Assume that $A$ is a quasi-attractor and let $x \in A$. We want to prove that $A=\operatorname{Ls} F^{n}(\{x\})$. Since $F^{n}(\{x\}) \subset A$ for all $n \geq 1$ and $A$ is a closed set then $\operatorname{Ls}^{n}(\{x\}) \subset A$. As in Proposition 2.3, $\operatorname{Ls} F^{n}(\{x\})$ is a forward invariant closed set and thus, by the minimality of $A$, $\operatorname{Ls} F^{n}(\{x\})=A$ and $x \in \mathcal{B}_{p}^{*}(A)$. Moreover, the same argument also proves that $\operatorname{Ls} F^{n}(K)=A$ for all non-empty compact set $K \subset A$. In fact, considering that Proposition 2.3 implies that

$$
\overline{\Gamma(y)}=\Gamma(y) \cup A \quad \text { for all } y \in \mathcal{B}_{p}^{*}(A),
$$

for all $z \in \overline{\Gamma(y)}$ it holds that if $z \in A$ then $\operatorname{Ls}^{n}(\{z\})=A$ and if $z \in \Gamma(y)$ then $\operatorname{Ls} F^{n}(\{z\}) \subset$ $\operatorname{Ls} F^{n}(\{y\})=A$ and from the above arguments $\operatorname{Ls} F^{n}(\{z\})=A$. Therefore the closure of $\Gamma(y)$ is contained in $\subset \mathcal{B}_{p}^{*}(A)$ for all $y \in \mathcal{B}_{p}^{*}(A)$.

Suppose now that $A \subset \mathcal{B}_{p}^{*}(A)$. Hence $A=\operatorname{Ls} F^{n}(\{x\})$ for all $x \in A$. In particular, $A$ is a forward minimal set and by Proposition 2.3 also is a forward invariant set. Thus $A$ is a forward invariant minimal set, that is, a quasi-attractor.

Finally we will proof the last conclusion. By the first conclusion, it suffices to show that if $A$ is an strict attractor then $\mathcal{B}_{p}^{*}(A) \subset \mathcal{B}_{p}(A)$. To accomplish this, let us consider $x \in \mathcal{B}_{p}^{*}(A)$ and open sets $U, V$ such that $A \subset U$ and $A \cap V \neq \emptyset$. Being $A$ a pointwise attractor there is an open neighborhood $W$ of $A$ so that $F(\{z\}) \rightarrow A$ in the Vietoris topology for all $z \in W$. Without loss of generality, we can assume that $U \subset W$. Since $A=\operatorname{LsF}^{n}(\{x\})$ then there exists $n_{1} \in \mathbb{N}$ such that $F^{n}(\{x\}) \subset U$ for all $n \geq n_{1}$. Then, for every $z \in F^{n_{1}}(\{x\})$ we have that $F^{n}(\{z\})$ converges to $A$ in the Vietoris topology and thus, by Lemma 2.2 , there is $n_{2}=n_{2}(z) \in \mathbb{N}$ so that

$$
F^{n}(\{z\}) \cap V \neq \emptyset \text { for all } n \geq n_{2} .
$$

Taking $n_{0}=\max \left\{n_{2}(z): z \in F^{n_{1}}(\{x\})\right\}$ we get that $F^{n}(\{x\}) \cap V \neq \emptyset$ for all $n \geq n_{0}$ and therefore, Lemma 2.2 implies that $x \in \mathcal{B}_{p}(A)$

We complete the proof of Theorem A by proving Conclusion (3).

Proposition 2.5. If $A$ is a strict attractor, then

(1) $F^{n}(K) \rightarrow A$ in the Vietoris topology for all compact sets $K \subset \mathcal{B}(A)$;

(2) $A$ is a pointwise attractor and $\mathcal{B}(A)=\mathcal{B}_{p}(A)$. 
Proof. The first conclusion is a consequence of Lemma 2.1. Indeed, given any compact set $K$ in $\mathcal{B}(A)$, by compactness we can find open neighborhoods $U_{1}, \ldots, U_{s}$ of $A$ such that $K \subset U_{1} \cup \cdots \cup U_{s}$ and $F^{n}(S) \rightarrow A$ for any compact set $S$ in $U_{i}$, for all $i=1, \ldots, s$. By Lemma 2.1 there are compact sets $K_{i} \subset U_{i}$ for $i=1, \ldots, s$ such that $K=K_{1} \cup \cdots \cup K_{s}$. Then,

$$
F^{n}(K)=F^{n}\left(K_{1}\right) \cup \cdots \cup F^{n}\left(K_{s}\right)
$$

and thus $F^{n}(K)$ converges to $A$ in the Vietoris topology.

We will prove the second conclusion. By means of the first conclusion, $\mathcal{B}(A) \subset \mathcal{B}_{p}(A)$. Thus, since $\mathcal{B}(A)$ is an open set containing $A$ we get that $A$ is a pointwise attractor. To conclude, we will show that $\mathcal{B}_{p}(A) \subset \mathcal{B}(A)$. Given $x \in \mathcal{B}_{p}(A)$ we want to prove that $x$ belongs to $\mathcal{B}(A)$.

Claim 2.5.1. If there exists a neighborhood $V$ of $x$ such that $F^{n}(K) \rightarrow A$ in the Vietoris topology for all non-empty compact sets $K \subset V$ then $x \in \mathcal{B}(A)$.

Proof. Since $A$ is an attractor there exists a neighborhood $U_{0}$ of $A$ such that $F^{n}(S) \rightarrow A$ for all compact sets $S$ in $U_{0}$. Take $U=U_{0} \cup V$. Clearly, $U$ is a neighborhood of $A$ and $x \in U$. On the other hand, by Lemma 2.1, any compact set $K$ in $U$ can be written as the union of two compact sets $K_{0}$ and $K_{1}$ contained in $U_{0}$ and $V$ respectively. Now, since $F^{n}(K)=F^{n}\left(K_{0}\right) \cup F^{n}\left(K_{1}\right)$ it follows that $F^{n}(K)$ converges to $A$ for all non-empty compact sets $K$ in the neighborhood $U$ of $A$. This implies that $x \in \mathcal{B}(A)$.

Now, we will get a neighborhood $V$ of $x$ in the assumptions of the above claim. Since $\mathcal{B}_{p}(A)$ is an open neighborhood of $A$ and $x \in \mathcal{B}(A)$, we get $m \in \mathbb{N}$ such that $F^{m}(\{x\}) \subset \mathcal{B}(A)$. Equivalently,

$$
f_{\omega_{m}} \circ \cdots \circ f_{\omega_{1}}(x) \in \mathcal{B}(A) \text { for all } \omega_{i} \in\{1, \ldots, k\} \text { for } i=1, \ldots, m \text {. }
$$

By the continuity of the generators $f_{1}, \ldots, f_{k}$ of the IFS, we get an open set $V$ such that $x \in V$ and

$$
f_{\omega_{m}} \circ \cdots \circ f_{\omega_{1}}(V) \subset \mathcal{B}(A) \text { for all } \omega_{i} \in\{1, \ldots, k\} \text { for } i=1, \ldots, m .
$$

In particular, for every compact set $K$ in $V$ it holds that $F^{m}(K) \subset \mathcal{B}(A)$ and thus, by the first conclusion, $F^{n}(K)$ converges to $A$. Finally Claim 2.5.1 implies that $x \in \mathcal{B}(A)$ as we wanted to show. This completes the proof.

To end this section we will prove Conclusion (2) of Theorem B.

Proposition 2.6. Consider $\omega \in \Omega$ and $x \in \mathcal{B}_{p}^{*}(A)$. Then the following statements are equivalent:

(1) $A \subset \overline{O_{\omega}^{+}(x)}$;

(2) $\lim _{n \rightarrow \infty} \overline{\left\{f_{\omega}^{m}(x): m \geq n\right\}}=A$ in the Vietoris topology;

(3) $A=\bigcap_{n \geq 1} \overline{\left\{f_{\omega}^{m}(x): m \geq n\right\}}$. 
Proof. According to Proposition 2.3, $A_{n}=\overline{\left\{f_{\omega}^{m}(x): m \geq n\right\}}$ is a compact set for all $n \in \mathbb{N}$. Moreover, $A_{n+1} \subset A_{n}$ and hence, by Lemma 2.2, if $A_{n} \rightarrow A$ in the Vietoris topology,

$$
A=\bigcap_{n \geq 1} A_{n} \subset A_{1}=\overline{O_{\omega}^{+}(x)}
$$

This proves (2) implies (1).

Reciprocally, let $U$ and $V$ be open sets such that $A \subset U$ and $V \cap A \neq \emptyset$. Since $x \in \mathcal{B}_{p}^{*}(A)$ then $A=\operatorname{Ls} F^{n}(\{x\})$ and thus there exists $n_{0} \in \mathbb{N}$ such that

$$
\bigcup_{n \geq n_{0}} F^{n}(\{x\}) \subset U
$$

In particular, the union of $A_{n}$ for $n \geq n_{0}$ is contained in $U$. Moreover, since $A \subset \overline{O_{\omega}^{+}(x)}$ we have $A_{n} \cap V \neq \emptyset$ for all $n$ large enough. Lemma 2.2 implies that $A_{n} \rightarrow A$ in the Vietoris topology completing the proof of (1) implies (2).

Finally, by Lemma 2.2, we have that (2) implies (3) and easily one can see that (3) implies (1) concluding the proof of the proposition

\section{Deterministic chaos game}

3.1. Equivalence. We will conclude Theorem B proving Conclusion (3).

Proposition 3.1. Let A be a quasi-attractor. Then the following statements are equivalent:

(1) there exists $\omega \in \Omega$ such that $A \subset \overline{O_{\omega}^{+}(x)}$ for all $x \in \mathcal{B}_{p}^{*}(A)$;

(2) $A \subset \overline{O_{\omega}^{+}(x)}$ for all disjunctive sequences $\omega \in \Omega$ and $x \in \mathcal{B}_{p}^{*}(A)$;

(3) there is $\Omega_{0} \subset \Omega$ with $\mathbb{P}\left(\Omega_{0}\right)=1$ such that

$$
A \subset \overline{O_{\omega}^{+}(x)} \text { for all } \omega \in \Omega_{0} \text { and } x \in \mathcal{B}_{p}^{*}(A) \text {. }
$$

Proof. It suffices to show that (1) implies (2). Let $x$ be a point in $\mathcal{B}_{p}^{*}(A)$. According to Proposition 2.3,

$$
K \stackrel{\text { def }}{=} \overline{\Gamma(x)} \subset \mathcal{B}_{p}^{*}(A)
$$

and it is a forward invariant compact set.

The following claim will be useful to prove the density of disjunctive fiberwise orbits, i.e, of fiberwise orbits driving by disjunctive sequences:

Claim 3.1.1. Let $Z$ be a forward invariant set such that $A \subset Z$. If for any non-empty open set $I \subset X$ with $A \cap I \neq \emptyset$, there is $f_{i_{s}} \circ \cdots \circ f_{i_{1}} \in \Gamma$ such that

$$
\text { for each } z \in Z \text { there is } t \in\{1, \ldots, s\} \text { so that } f_{i_{t}} \circ \cdots \circ f_{i_{1}}(z) \in I
$$

then

$$
A \subset \overline{O_{\omega}^{+}(x)} \text { for all disjunctive sequences } \omega \in \Omega \text { and } x \in Z \text {. }
$$


Proof. Consider any open set $I$ such that $A \cap I \neq \emptyset, x \in Z$ and a disjunctive sequence $\omega \in \Omega$. Using the fact that $\omega$ is a disjunctive sequence and that $Z$ is a forward invariant set we can choose $m \geq 1$ such that

$$
\left[\sigma^{m}(\omega)\right]_{j}=i_{j} \text { for } j=1, \ldots, s \text { and } z=f_{\omega}^{m}(x) \in Z \text {. }
$$

Hence, by assumption, there exists $t=t(z)$ such one has that $f_{\omega}^{m+t(z)}(x) \in I$ which proves the density on $A$ of the $\omega$-fiberwise orbit of $x$.

Notice that $F(K) \subset K$ and hence we can take as $Z=K$ in the above claim. Let $I$ be an open set so that $I \cap A \neq \emptyset$. By assumption, since $Z \subset \mathcal{B}_{p}(A)$, there exists a sequence $\omega \in \Omega$ such that for each point $z \in Z$ the $\omega$-fiberwise orbit of $z$ is dense in $A$. In particular, there is $n=n(z) \in \mathbb{N}$ such that

$$
\left\{f_{\omega}^{m}(z): m \leq n\right\} \cap I \neq \emptyset .
$$

By continuity of the generators $f_{1}, \ldots, f_{k}$ of the IFS, there exists an open neighborhood $V_{z}$ of $z$ such that

$$
\left\{f_{\omega}^{m}(y): m \leq n\right\} \cap I \neq \emptyset \text { for all } y \in V_{z} .
$$

Then, the compactness of $Z$ implies that we can extract open sets $V_{1}, \ldots, V_{r}$ and positive integers $n_{1}, \ldots, n_{r}$ such that $Z \subset V_{1} \cup \cdots \cup V_{r}$ and

$$
\left\{f_{\omega}^{m}(z): m \leq n_{i}\right\} \cap I \neq \emptyset \text { for all } z \in V_{i} \text { and } i=1, \ldots, r .
$$

Hence the assumptions of Claim 3.1.1 hold taking $f_{\omega_{s}} \circ \cdots \circ f_{\omega_{1}} \in \Gamma$ where $s=\max \left\{n_{i}\right.$ : $i=1, \ldots, r\}$. Therefore, since the initial point $x \in \mathcal{B}_{p}^{*}(A)$ belongs to $Z$, we conclude that any disjunctive fiberwise orbit of $x$ is dense in $A$ that completes the proof.

3.2. Necessary condition. We will prove Theorem E.

Proof of Theorem E. Clearly if there is a minimal orbital branch, i.e., $\omega=\omega_{1} \omega_{2} \cdots \in \Omega$ such that $O_{\omega}^{+}(x)$ is dense for all $x$, then the IFS is forward minimal.

We will assume that it is not backward minimal. Then, there exists a non-empty closed set $K \subset X$ such that $\emptyset \neq f^{-1}(K) \subset K \neq X$ for all $f \in \Gamma$. We can consider

$$
K_{n}^{-}=\bigcap_{i=1}^{n} f_{\omega_{1}}^{-1} \circ \cdots \circ f_{\omega_{i}}^{-1}(K)=f_{\omega_{1}}^{-1} \circ \cdots \circ f_{\omega_{n}}^{-1}(K) \text { and } K_{\omega}^{-}=\bigcap_{n=1}^{\infty} K_{n}^{-} .
$$

Hence $K_{n}^{-}$is a nested sequence of closed sets. By assumption of this theorem, the space $X$, where the IFS is defined, is a compact Hausdorff topological space. As a consequence, $K_{\omega}^{-}$is not empty and then for every $x \in K_{\omega}^{-}$we have that $O_{\omega}^{+}(x) \subset K$. Since $K$ is not equal to $X$ it follows that there exists a point $x \in X$ so that the $\omega$-fiberwise orbit of $x$ is not dense. But this is a contradiction and we conclude the proof.

As in the introduction we notified, an IFS is forward minimal if and only if every point has dense $\Gamma$-orbit. To complete the section we want to point out the following straightforward similar equivalent definition of backward minimality. 
Lemma 3.2. Consider an IFS of surjective continuous maps of a topological space X. Then the IFS is backward minimal if and only if $X=\overline{\Gamma^{-1}(x)}$ for all $x \in X$ where

$$
\Gamma^{-1}(x) \stackrel{\text { def }}{=}\{y \in X \text { : there exists } g \in \Gamma \text { such that } g(y)=x\} .
$$

3.3. Minimal IFSs of homeomorphisms of the circle. As consequence of Theorem E, we will obtain that the deterministic chaos game is totally characterized for forward minimal IFSs of homeomorphisms of the circle. Moreover, this characterization allows us to construct attractors of IFSs that is not renderable by the deterministic chaos game (counterexamples).

3.3.1. Characterization. In [12, Thm. A] it was proved that every forward and backward minimal IFS of preserving-orientation homeomorphisms of the circle is renderable by the deterministic chaos game. However, the assumption of preserving-orientation can be removed from this statement as we explain below.

The main tool in the proof of [12, Thm. A] was Antonov's Theorem [1] (see [12, Thm. 2.1]). This theorem is stated for preserving-orientation homeomorphisms of the circle. Supported in this result the authors showed a key lemma (see [12, Lem. 2.2]) to prove the above statement. In fact, in this lemma, by means of Antonov's result, is the unique point in the proof where the preserving-orientation assumption is used. This lemma can be improved removing the preserving orientation assumption by two different ways. The first is observing that in fact, this assumption is not necessarily in the original proof of Antonov as easily one can follow from the argument described in [21, proof of Theorem 2]. Another way is to use the recently generalization of Antonov's result [29, Thm. D] instead the key lemma above mentioned.

Proof of Corollary I. From above, every forward and backward minimal IFS of homeomorphisms of the circle is renderable by the deterministic chaos game. That is, (3) implies (1). On the other hand, (1) implies (3) follows from Theorem E. Finally, to complete the proof of the corollary it suffices to note that according to Theorem B, (1) and (2) are equivalent.

3.3.2. Counterexample. We will prove now Corollary II. As in the introduction we mentioned, for ordinary dynamical systems, the minimality of a map $T$ is equivalent to that of $T^{-1}$. Nevertheless this is not the case for dynamical systems with several maps as Kleptsyn and Nalskii pointed at [23, pg. 271]. However, they omitted to include these examples of forward but not backward minimal IFSs. Hence, to provide a complete proof of Corollary II we will show that indeed such IFSs of homeomorphisms of $S^{1}$ can be constructed.

a) Forward but not backward minimal IFSs on the circle: Consider a group G of homeomorphisms of the circle. Then, there can occur only one of the following three options [32, 20]:

(1) there is a finite G-orbit,

(2) every G-orbit is dense on the circle, or

(3) there is a unique G-invariant minimal Cantor set. 
By a $G$-orbit we understand the action of $G$ at a point $x \in S^{1}$. That is the set of points $G(x)=\{g(x): g \in G\}$. If $G(x)$ has finitely many different elements then it is called finite orbit while if its closure is $S^{1}$, it say dense orbit. The Cantor set $K$ in the above third conclusion is usually called exceptional minimal set. This set is G-invariant and minimal, that is,

$$
g(K)=K \text { for all } g \in G \text { and } K=\overline{G(x)} \text { for all } x \in K .
$$

Notice that these properties are the same to say that $K$ is minimal regarding to the inclusion of $G$-invariant closed sets. The following proposition is stated in [32, Exer. 2.1.5]. For completeness, we include the proof.

Proposition 3.3. There exists a finitely generated group $G$ of homeomorphisms of $S^{1}$ admitting an exceptional minimal set $K$ such that the G-orbit of every point of $S^{1} \backslash K$ is dense in $S^{1}$.

Proof. Let $f$ a homeomorphism of the circle with a minimal exceptional set $K$ and such that there is only one class of gaps, which means that for every gaps $I, J$, there exists $n$ in $\mathbb{Z}$ such that $f^{n}(I)=J$. For instance, the classic Denjoy map. Let $I_{0}$ be a gap of $K$. Let $u: I_{0} \rightarrow \mathbb{R}$ be a homeomorphism, $\tilde{f}_{1}$ and $\tilde{f_{2}}$ be respectively the translations $x \mapsto x+1$ and $x \mapsto x+\sqrt{2}$ on $\mathbb{R}$, and let us set define two homeomorphisms $f_{1}$ and $f_{2}$ of $S^{1}$ by $f_{i}=u^{-1} \tilde{f}_{i} u$ on $I_{0}, f_{i}=\mathrm{id}$ on $S^{1} \backslash I_{0}$. We claim that the group $G$ generated by $f, f_{1}$ and $f_{2}$ satisfies the required properties. Obviously, $K$ is also the minimal exceptional of $G$ since $\left.f_{i}\right|_{K}=i d$. On the other hand, the subgroup $H$ generated by $f_{1}$ and $f_{2}$ leaves the gap $I_{0}$ invariant and acts minimally on it since the group generated by $\tilde{f}_{1}$ and $\tilde{f}_{2}$ acts minimally on $\mathbb{R}$. Hence, let $x$ be in $S^{1} \backslash K$ and $I$ be an interval of the circle. Since there is only one class of gaps one can find $m$ and $n$ in $\mathbb{Z}$ such $f^{m}(x) \in I_{0}$ and $f^{n}(I) \cap I_{0} \neq \emptyset$. Next, by minimality of the action of $H$ on $I_{0}$, one can find $h$ in $H$ such that $h\left(f^{m}(x)\right) \in f^{n}(I)$. Thus, the element $g=f^{-n} h f^{m}$ of $G$ sends $x$ into $I$. Since $I$ is arbitrary by $G$, the orbit of $x$ by $G$ is dense.

We say that a subset $\mathscr{F}$ of a group $G$ is a symmetric generating system of $G$ if $G$ is generated by $\mathscr{F}$ as a semigroup. Moreover, we ask that if $f \in \mathscr{F}$ then also $f^{-1} \in \mathcal{F}$. Hence, we can see the action of the group as a symmetric IFS generated by $\mathscr{F}$.

Remark 3.4. Let $\mathscr{F}$ be a finite symmetric generating system of the group G given in Proposition 3.3. Hence, from the above observation, it follows that the exceptional minimal set $K$ is the unique quasiattractor of the symmetric IFS generated by $\mathscr{F}$ and it holds that $\mathcal{B}_{p}^{*}(K)=K$. This provides an example of a quasi-attractor of a non-minimal IFS which cannot be a pointwise attractor.

We will use the following:

Lemma 3.5. Let $G$ and $K$ be as in Proposition 3.3. Then the closed subsets of $S^{1}$ which are invariant by $G$ are $\emptyset, K$ and $S^{1}$.

Proof. Let $B$ be a closed subset of $S^{1}$ invariant by $G$. If $B \neq \emptyset$, then $K \subset B$ by minimality of $K$, and if $B \neq K$, it means that $B$ contains a point $x$ in $S^{1} \backslash K$, and by invariance, $B$ contains the orbit of $x$ by $G$ which is dense, hence $B=S^{1}$. 
On the other hand, any two Cantor set are homemorphic. In fact, if $K_{I}$ and $K_{J}$ are two Cantor sets in an interval $I$ and $J$ respectively, there exists a homeomorphisms $g: I \rightarrow J$ so that $g\left(K_{I}\right)=K_{J}$ (see for instance [16]). Hence given any Cantor set $K$ in $S^{1}$ one can find a homeomorphisms $h$ of $S^{1}$ so that $h(K)$ is strictly contained in $K$ (or $h(K)$ strictly contains $K$ ).

Proposition 3.6. Let $G$ and $K$ be as in Proposition 3.3 and $f_{1}, \ldots, f_{n}$ be a symmetric system of generators of $G$. Consider any homeomorphisms $h$ of $S^{1}$ such that $h(K)$ strictly contains $K$. Then the IFS generated by $f_{1}, \ldots, f_{n}, h$ is forward minimal but not backward minimal.

Proof. Let $K_{1} \stackrel{\text { def }}{=} h(K)$. By assumption $K \subsetneq K_{1}$. We claim that the IFS generated by $f_{1}, \ldots, f_{n}, h$ is forward minimal but not backward minimal.

- The IFS is not backward minimal: since $K$ is invariant by the group $G$,

$$
f_{i}^{-1}(K)=K \text { for } i=1, \ldots, n .
$$

We also have $h^{-1}(K) \subset h^{-1}\left(K_{1}\right)=K$. Thus, $K$ is forward invariant by $f_{1}^{-1}, \ldots, f_{n}^{-1}, h^{-1}$ and so the IFS is not backward minimal.

- The IFS is forward minimal: let $B \subset S^{1}$ be a forward invariant by $f_{1}, \ldots, f_{n}, h$ closed set. In particular $B$ is invariant by $G$, hence $B \in\left\{\emptyset, K, S^{1}\right\}$ by Lemma 3.5. Moreover $B \neq K$ since $K$ is not invariant by $h$ (otherwise $K_{1}=h(K)=h(B) \subset B=K$ but $K_{1}$ strictly contains $K$ ). So, $B \in\left\{\emptyset, S^{1}\right\}$, which means that the IFS is forward minimal.

b) Strict attractors: To complete the proof of Corollary II we need to show that $S^{1}$ is a strict attractor of the IFS generated by $f_{1}, \ldots, f_{n}, h$ in Proposition 3.6. We infer this from the next result.

We say that an IFS generated by a family $\mathscr{F}$ of continuous maps of $X$ is quasi-symmetric if there is $f \in \mathscr{F}$ so that it inverse map $f^{-1} \in \mathscr{F}$.

Proposition 3.7. Consider a minimal quasi-symmetric IFS on a compact connected Hausdorff space $X$. Then $X$ is an strict attractor of this IFS.

Before to prove the above proposition we need the following Lemma (c.f. [29, Lemma 4.15]). Again, for completeness, we include the proof.

Lemma 3.8. Consider a minimal IFS generated by a family $\mathscr{F}$ of continuous maps of a connected Hausdorff topological space X. Then the IFS generated by $\mathscr{F}^{2}=\{f \circ g: f, g \in \mathscr{F}\}$ is also minimal.

Proof. Throughout the proof, we extend the Hutchinson operator $F=F_{\mathscr{F}}$ to the hyperspace of non-empty closed sets. We want to prove that if $B$ be a non-empty closed subset of $X$ so that $F^{2}(B) \subset B$ then $B=X$. Notice that

$$
B^{\prime} \stackrel{\text { def }}{=} B \cup F(B) \quad \text { and } \quad B^{\prime \prime} \stackrel{\text { def }}{=} B \cap F(B)
$$

are both forward invariant set, i.e. $F\left(B^{\prime}\right) \subset B^{\prime}$ and $F\left(B^{\prime \prime}\right) \subset B^{\prime \prime}$. By the minimality of the IFS generated by $\mathscr{F}$ it follows that $B^{\prime}=X$. Hence, since $X$ is a connected space and both $B$ and $F(B)$ are closed we get that $B^{\prime \prime} \neq \emptyset$. Thus, again by the minimality we have that $B^{\prime \prime}=X$ and therefore $B=X$. 
Proof of Proposition 3.7. Let $K$ be a compact set of $X$. We want to show that $F^{n}(K) \rightarrow X$ in the Vietoris topology. The first observation is that, since the IFS is quasi-symmetric then $K \subset F^{2}(K)$. Then,

$$
\left(F^{2}\right)^{n}(K) \subset\left(F^{2}\right)^{n+1}(K) \text { for all } n \geq 1 .
$$

By Lemma 3.8, the IFS generated by $\mathscr{F}^{2}$ is also minimal and thus, for every open set $V$ of $X$ there is $n_{0} \in \mathbb{N}$ such that $\left(F^{2}\right)^{n_{0}}(K) \cap V \neq \emptyset$. So, by the monotonicity of this sequence, $\left(F^{2}\right)^{n}(K) \cap V \neq \emptyset$ for all $n \geq n_{0}$. Thus, according to Lemma 2.2, we have that $\left(F^{2}\right)^{n}(K) \rightarrow X$ in the Vietoris topology. By means of the continuity of the Hutchinson operator $F$ and since $X$ is a self-similar set we also have that $F^{2 n+1}(K) \rightarrow X$. Thus, we conclude that $F^{n}(K) \rightarrow X$.

We want to remark that in the case of the homeomorphisms of the circle we have an stronger result:

Proposition 3.9. Let $f_{1}, \ldots f_{k}$ homeomorphisms of $S^{1}$ without a common invariant probability measure, and such that the IFS generated by them is minimal. Then $S^{1}$ is a strict attractor for this IFS.

Proof. Let $x$ in $S^{1}$, and let $\mu_{n}$ be the law of $f_{\omega}^{n}(x)=f_{\omega_{n}} \circ \cdots \circ f_{\omega_{1}}(x)$, where $\omega_{1}, \ldots, \omega_{n}$ are chosen independently and uniformly on $\{1, \ldots, k\}$. By $\left[29\right.$, Cor. 2.6], the sequence $\left(\mu_{n}\right)_{n \in \mathbb{N}}$ converges weakly as $n \rightarrow \infty$ to the unique stationary probability measure $\mu$ of the system, i.e. to the self-similar measure

$$
\mu=\frac{1}{k}\left(\left(f_{1}\right)_{*} \mu+\cdots+\left(f_{k}\right)_{*} \mu\right) .
$$

Moreover, this measure $\mu$ has total support because its topological support is invariant by $f_{1}, \ldots, f_{k}$. Consequently, for any interval $I$ of the circle, $\mu(I)>0$ and so $\mu_{n}(I)>0$ for $n$ large enough. Since we clearly have that $\operatorname{supp}\left(\mu_{n}\right) \subset F^{n}(\{x\})$, we deduce that $F^{n}(\{x\}) \cap I \neq \emptyset$ for all $n$ sufficient large, and hence we conclude by Lemma 2.2 that $S^{1}$ is an atractor.

3.4. Sufficient conditions. In what follows, $A$ denotes a quasi-attractor.

3.4.1. Well-fibred attractors. We start studying the relation between strongly-fibred and wellfibred quasi-attractors.

Proposition 3.10. If $A$ is strongly-fibred then it is well-fibred. Moreover, if in addition $A$ is a strict attractor then for every compact set $K$ in $\mathcal{B}(A)$ and every open set $U$ so that $A \cap U \neq \emptyset$ there exists $g \subset \Gamma$ such that $g(K) \subset U$.

Proof. Consider a compact set $K$ in $A$ and let $U$ be any open set such that $A \cap U \neq \emptyset$. Since $A$ is strongly-fibred, we get $\omega \in \Omega$ such that

$$
A_{\omega}=\bigcap_{n=1}^{\infty} f_{\omega_{1}} \circ \cdots \circ f_{\omega_{n}}(A) \subset U .
$$

Notice that since $f_{i}(A) \subset A$ for $i=1, \ldots, k$ then $f_{\omega_{1}} \circ \cdots \circ f_{\omega_{n}}(A)$ is a nested sequence of compact sets and thus, for $n$ large enough, $f_{\omega_{1}} \circ \cdots \circ f_{\omega_{n}}(A) \subset U$. In particular, taking $h=f_{\omega_{1}} \circ \cdots \circ f_{\omega_{n}} \in \Gamma$ we have that $h(K) \subset U$. This proves that $A$ is well-fibred. 
We will assume now that $A$ is a strict attractor and consider $K$ in $\mathcal{B}(A)$. As above we have that $h(A) \subset U$. We claim that there exists a neighborhood $V$ of $A$ such that $h(V) \subset U$. Indeed, it suffices to note that $h$ is a continuous map and hence $h^{-1}(U)$ is an open set containing the compact set $A$. Since $A$ is a strict attractor, $F^{n}(K) \rightarrow A$ in the Vietoris topology and in particular, there is $f \in \Gamma$ such that $f(K) \subset V$. Thus, taking $g=h \circ f \in \Gamma$, it follows that $g(K) \subset h(V) \subset U$.

Remark 3.11. If $A$ is strongly fibred we have proved that one can contract any compact set in $A$. In particular we can contract $A$ and this implies that there exists some generator $f_{i}$ such that $f_{i}(A) \neq A$.

Now, we give an example of an IFS defined on $S^{1}$ whose unique strict attractor is the whole space (that is the IFS is minimal) and it is well-fibred but not strongly-fibred. This example shows that these two properties are not equivalent. See also Corollary 3.17 at the end of this subsection.

Example 3.12. Consider the IFS generated by two diffeomorphisms $g_{1}, g_{2}$, where $g_{1}$ is rotation with irrational rotation number and $g_{2}$ is an orientation preserving diffeomorphism with a unique fixed point $p$ such that $\operatorname{Dg}_{2}(p)=1$ and $\alpha$-limit set and $\omega$-limit set of each point $q \in S^{1}$ is equal to $\{p\}$. Clearly, the IFS acts minimally on $S^{1}$ and have no common invariant measure thus $A=S^{1}$ is the attractor. Since $g_{1}$ and $g_{2}$ map $S^{1}$ onto itself, it follows that for each $\omega \in \Omega$, the fiber $A_{\omega}=S^{1}$. This implies that $S^{1}$ is not strongly-fibred, but it still well-fibred. Indeed, let $K$ be any compact set so that $K \neq S^{1}$. Then, there is an open arc J of $S^{1}$ which is not dense in $S^{1}$ such that $K \subset J$. If J contains the fixed point $p$, there is an integer $n$ such that $g_{1}^{n}(J)$ does not contain $p$. So, without less of generality, we may assume that $p \notin J$. Now, it is easy to see that $g_{2}^{k}(J)$ tends to $p$ as $k \rightarrow \infty$. This implies that $A=S^{1}$ is well-fibred.

Above example is based on the fact that $A$ satisfies that $f_{i}(A)=A$ for all $i=1, \ldots, k$. The above proposition and the following show that if $f_{i}(A)$ is not equals to $A$ for some generator $f_{i}$ then both properties are equivalent.

Proposition 3.13. If $A$ is well-fibred and $f_{i}(A) \neq A$ for some $i \in\{1, \ldots, k\}$ then $A$ is strongly-fibred.

Proof. First of all note that it suffices to prove that for any open set $U$ with $U \cap A \neq \emptyset$, there is $h \in \Gamma$ so that $h(A) \subset U$. To this end, notice that since $A$ is a quasi-attractor then the action of $\Gamma$ restricted to $A$ is minimal. Then, there exist $h_{1}, \ldots, h_{m} \in \Gamma$ so that $A \subset h_{1}^{-1}(U) \cup \cdots \cup h_{m}^{-1}(U)$. On the other hand, by assumption, there is $i \in\{1, \ldots, k\}$ such that $f_{i}(A) \neq A$. Hence $f_{i}(A)$ is a compact set strictly contained in $A$ and since $A$ is well-fibred there exist $g \in \Gamma$ and $j \in\{1, \ldots, m\}$ such that $g\left(f_{i}(A)\right) \subset h_{j}^{-1}(U)$. Thus, taking $h=h_{j} \circ g \circ f_{i} \in \Gamma$, it follows that $h(A) \subset U$ concluding the proof.

In order to proof Theorem $C$, we need a lemma (compare with Claim 3.1.1). Here we understand $f_{i_{t}} \circ \cdots \circ f_{i_{1}}$ for $t=0$ as the identity map.

Lemma 3.14. If for any non-empty open set $I \subset X$ with $A \cap I \neq \emptyset$, there exist a neighborhood $Z$ of $A$ and $f_{i_{s}} \circ \cdots \circ f_{i_{1}} \in \Gamma$ such that

$$
\text { for each } z \in Z \text { there is } t \in\{0, \ldots, s\} \text { so that } f_{i_{t}} \circ \cdots \circ f_{i_{1}}(z) \in I
$$


then

$$
A \subset \overline{O_{\omega}^{+}(x)} \text { for all disjunctive sequences } \omega \in \Omega \text { and } x \in \mathcal{B}_{p}^{*}(A) \text {. }
$$

Proof. Consider $x \in \mathcal{B}_{p}^{*}(A)$, disjunctive sequence $\omega \in \Omega$ and any open set $I$ such that $A \cap I \neq \emptyset$. Being $Z$ a neighborhood of $A$ and $\operatorname{Ls} F^{n}(\{x\})=A$ we can choose $m \geq 1$ such that

$$
\left[\sigma^{m}(\omega)\right]_{j}=i_{j} \text { for } j=1, \ldots, s \text { and } z=f_{\omega}^{m}(x) \in Z .
$$

Hence, by assumption, there exists $t=t(z)$ such one has that $f_{\omega}^{m+t}(x) \in I$ which proves the density on $A$ of the $\omega$-fiberwise orbit of $x$.

The following result proves the first part in Theorem C.

Proposition 3.15. A well-fibred quasi-attractor $A$ is renderable by the deterministic chaos game.

Proof. In order to apply Lemma 3.14, we consider any non-empty open set $I$ with $I_{A} \stackrel{\text { def }}{=} A \cap I \neq$ $\emptyset$. Hence, $K=A \backslash I_{A}$ is a compact set so that $K \neq A$. Since $A$ is a quasi-attractor, the action of $\Gamma$ restrict to $A$ is minimal and thus, there exist $h_{1}, \ldots, h_{m} \in \Gamma$ such that $A \subset h_{1}^{-1}(I) \cup \cdots \cup h_{m}^{-1}(I)$. On the other hand, since $A$ is well-fibred, there exist $i \in\{1, \ldots, m\}$ and $g \in \Gamma$ such that $g(K) \subset h_{i}^{-1}(I)$. Take $h=h_{i} \circ g$. By continuity of the generators we find an open set $U$ with $K \subset U$ such that $h(U) \subset I$. Take, $Z=U \cup I$ and $f_{i_{s}} \circ \cdots \circ f_{i_{1}}=h \in \Gamma$. Clearly, $Z$ is open with $A=K \cup I_{A} \subset U \cup I=Z$ and for every $z \in Z$, there is $t \in\{0, s\}$ such that $f_{i_{t}} \circ \cdots \circ f_{i_{1}}(z) \in I$. Lemma 3.14 implies $A$ is renderable by the deterministic chaos game.

Now, we conclude the proof of Theorem C.

Proposition 3.16. Consider a well-fibred forward minimal IFS generated by continuous maps of a compact Hausdorff topological space A. Assume the IFS is either, strongly-fibred or invertible (its generators are homeomorphisms). Then

$$
\Omega \times A=\overline{\left\{\Phi^{n}(\omega, x): n \in \mathbb{N}\right\}} \text { for all disjunctive sequences } \omega \text { and } x \in A .
$$

Proof. Let $\omega \in \Omega$ be a disjunctive sequence and consider $x \in A$. We want to show that $(\omega, x)$ has dense orbit in $\Omega \times A$ under the skew-product $\Phi$. In order to prove this, let $C_{\alpha}^{+} \times I$ be a basic open set of $\Omega \times A$. That is, $C_{\alpha}^{+}$is a cylinder in $\Omega$ around of a finite word $\alpha=\alpha_{1} \ldots \alpha_{\ell}$ and $I$ is an open set in $A$. In fact, we can assume that $I$ is not equal to the whole space. It suffices to prove that there exists an iterated by $\Phi$ of $(\omega, x)$ that belongs to $C_{\alpha}^{+} \times I$. To do this, similarly as in the previous proposition, we use the forward minimality of $\Gamma$ on $A$ to find maps $h_{1}, \ldots, h_{m} \in \Gamma$ such that $A=h_{1}^{-1}(I) \cup \cdots \cup h_{m}^{-1}(I)$. Set $f=f_{\alpha_{\ell}} \circ \cdots \circ f_{\alpha_{1}} \in \Gamma$.

Assume first that the IFS is strongly-fibred. Then there exists a generator $f_{i}$ such that $K=f_{i}(A) \neq A$. By Proposition 3.10, the IFS is well-fibred and thus we find $g \in \Gamma$ such that $g(K) \subset h_{\ell}^{-1}(I)$ for some $\ell \in\{1, \ldots, m\}$. Hence, $h(K) \subset I$ where $h=h_{\ell} \circ g$. Let $f \circ h \circ f_{i}=f_{i_{s}} \circ \cdots \circ f_{i_{1}}$. Since $\omega \in \Omega$ is a disjunctive sequence, we can choose $m \geq 1$ such that

$$
\left[\sigma^{m}(\omega)\right]_{j}=i_{j} \text { for } j=1, \ldots, s .
$$


Set $z=f_{\omega}^{m}(x)$. Then, $h \circ f_{i}(z) \in I$. Moreover,

$$
\Phi^{m+t}(\omega, x)=\Phi^{t}\left(\sigma^{m}(\omega), z\right)=\left(\sigma^{m+t}(\omega), h \circ f_{i}(z)\right) \in C_{\alpha}^{+} \times I
$$

where $t=1+|h|$ being $|h|$ the length of $h$ with respect to $\mathscr{F}=\left\{f_{1}, \ldots, f_{k}\right\}$.

Now, assume that the well-fibred forward minimal IFS is also invertible. Hence $f$ is a homeomorphism of $A$ and thus $\emptyset \neq f(I) \neq A$ is an open set. Let $K=A \backslash f(I)$. Notice that $K$ is a non-empty compact set different of $A$ and by means of the "contractibility" of the IFS we get $g \in \Gamma$ so that $h(K) \subset I$ where $h=h_{\ell} \circ g$ for some $\ell \in\{1, \ldots, m\}$. Let $f \circ h \circ f=f_{i_{s}} \circ \cdots \circ f_{i_{1}}$. Similar as above, since $\omega$ is a disjunctive sequence we choose $m \geq 1$ satisfying (3) and denote $z=f_{\omega}^{m}(x)$. If $z \in I, \Phi^{m}(\omega, x)=\left(\sigma^{m}(\omega), z\right) \in C_{\alpha}^{+} \times I$. Otherwise, $f(z) \in K$ and then $h \circ f(z) \in I$ and thus

$$
\Phi^{m+t}(\omega, x)=\Phi^{t}\left(\sigma^{m}(\omega), z\right)=\left(\sigma^{m+t}(\omega), h \circ f(z)\right) \in C_{\alpha}^{+} \times I
$$

where $t=|f|+|h|$ being $|f|$ and $|h|$ the length of $f$ and $h$ respectively.

We end this subsection showing a broad family of IFSs with a well-fibred quasi-attractor which are not strongly-fibred. Notice that this family contains the IFS of Example 3.12.

Corollary 3.17. Consider a forward and backward minimal IFS of homeomorphisms of a metric space $X$ and assume that there is a map $h$ in the semigroup $\Gamma$ generated by these maps with exactly two fixed points, one attracting and one repelling. Then $X$ is a well-fibred quasi-attractor and consequently is renderable by the deterministic chaos game.

Proof. The forward minimality implies that $X$ is a quasi-attractor. Consider now any compact set $K \subset X$ such that $K \neq X$. By the backward minimality there exist $T_{1}, \ldots, T_{s} \in \Gamma$ such that

$$
X=\bigcup_{i=1}^{s} T_{i}(X \backslash K)
$$

Let $p$ and $q$ be, respectively, the attracting and the repelling fixed points of $h$. Then there is $i \in\{1, \ldots, s\}$ so that $q \in T_{i}(X \backslash K)$. Therefore, $q \notin T_{i}(K)$ and then the diameter of $h^{n} \circ T_{i}(U)$ converges to zero. This shows that the action is well-fibred and completes the proof.

3.4.2. Quasi-attractors of symmetric IFSs. We will proof Theorem D. This theorem extends [19, Thm. 3.3] for compact Hausdorff topological spaces.

Proposition 3.18. If $A$ is a quasi-attractor of a symmetric IFS on a Hausdorff topological space then it is renderable by the deterministic chaos game.

Proof. We will use Lemma 3.14. To accomplish this, let $I$ be an open set so that $A \cap I \neq \emptyset$. By the minimality of the action of $\Gamma$ restricted to $A$, there are $h_{1}, \ldots, h_{m} \in \Gamma$ so that $A \subset$ $h_{1}^{-1}(I) \cup \cdots \cup h_{m}^{-1}(I)$. Set $Z$ be the union of these open sets. Since the IFS has a symmetric system of generators $\mathscr{F}=\left\{f_{1}, \ldots, f_{k}\right\}$, we can write

$$
f_{i_{s}} \circ \cdots \circ f_{i_{1}}=h_{m}^{-1} \circ h_{m} \circ \cdots \circ h_{2}^{-1} \circ h_{2} \circ h_{1}^{-1} \circ h_{1} .
$$


Hence,

$$
Z \subset \bigcup_{j=1}^{t} f_{i_{1}}^{-1} \circ \cdots \circ f_{i_{j}}^{-1}(I) .
$$

This implies that for each $z \in Z$, there is $t \in\{1, \ldots, s\}$ so that $f_{i_{t}} \circ \cdots \circ f_{i_{1}}(z) \in I$. Thus, by Lemma 3.14, $A$ is renderable by the deterministic chaos game.

To end this subsection, we give an example of a quasi-attractor of an symmetric IFS on the torus $\mathbb{T}^{2}$ which is not neither well-fibred nor a quasi-attractor of a non-expansive IFS nor have a minimal map.

Example 3.19. Let $f: \mathbb{T}^{2} \rightarrow \mathbb{T}^{2}$ be a generalized north-south pole diffeomorphism on the torus $\mathbb{T}^{2}$. By this we mean that the non-wandering set of $f, \Omega(f)$, consists of one fixed source, $q$, one fixed sink, $p$, and saddle type periodic orbits. Let $S$ be the set of all the saddle type periodic points of $f$. For simplicity we assume that $S$ consists of two saddle points so that $\mathcal{W}=W^{S}(S) \cup W^{u}(S) \cup\{p, q\}$ consists of forth circles: two disjoint circles following the meridian direction other two disjoint circles following the parallel directions. For every $x \in \mathbb{T}^{2} \backslash \mathcal{W}$ it holds that $f^{n}(x) \rightarrow p$ and $f^{-n}(x) \rightarrow q$ as $n \rightarrow \infty$. On the other hand, consider a translation $R_{\lambda}: \mathbb{T}^{2} \rightarrow \mathbb{T}^{2}, R_{\lambda}(x, y)=\left(x+\lambda_{1}, y+\lambda_{2}\right)$, where $\lambda=\left(\lambda_{1}, \lambda_{2}\right)$ is an irrational vector, i.e., $\lambda_{i} \in \mathbb{R} \backslash \mathbb{Q}$ for $i=1$, 2. Since the IFS generated by $f, f^{-1}, R_{\lambda}, R_{\lambda}^{-1}$ on $\mathbb{T}^{2}$ has minimal elements, according to [12, Prop. 1], it is renderable by the deterministic chaos game. Moreover, it is not difficult to see that this IFS is $C^{1}$-robustly minimal, i.e., the minimality persists under small $C^{1}$-perturbations on the generators (indeed, easily one can construct a "blending region" around the attracting fixed point and then apply [13, Thm. 6.3]). Thus, there is a rational vector $\alpha$ closed to $\lambda$ so that the IFS generated by $f, f^{-1}, R_{\alpha}, R_{\alpha}^{-1}$ acts minimally on $\mathbb{T}^{2}$. By Theorem $D$, this IFS is renderable by the deterministic chaos game. Clearly, it does not contain any minimal element and it is not a non-expansive IFS. Also, it is not well-fibred (indeed, it suffices to consider a compact neighborhood of a circle that contains $p$ and the unstable manifold of one saddle).

\section{Appendix A.}

In this appendix we extend the results due to Bransley, Leśniak and Rypka, [7, 27] on the probabilistic and the deterministic chaos game for attractors of IFSs to the general case of quasi-attractors. The proofs basically follow the same ideas of $[7,27]$ with some minor modifications and improvements.

\section{On Bransley, Leśniak and Rypka probabilistic chaos game for quasi-attractors:}

Theorem. Every first-countable quasi-attractor of an IFS of continuous maps of a Hausdorff topological space is renderable by the probabilistic chaos game.

Proof. Let $x_{0}$ be a point of $\mathcal{B}_{p}^{*}(A)$ and let $U$ an open subset of $A$. We want to prove that the event

$$
E=E\left(x_{0}, U\right) \stackrel{\text { def }}{=}\left\{\omega \in \Omega: O_{\omega}^{+}\left(x_{0}\right) \cap U \neq \emptyset\right\}
$$

has probability 1 . Since $A$ is a minimal forward invariant set, for any $x$ in $A$ we can find a finite sequence $i_{1}, \ldots, i_{m}$ such that $f_{i_{m}} \circ \cdots \circ f_{i_{1}}(x)$ belongs to $U$. Then, by using the compactness 
of $A$ and the continuity of the generators we can actually find an integer $m_{0}$ and functions $i_{1}, \ldots, i_{m_{0}}$ from a neighborhood $V$ of $A$ into $\{1, \ldots k\}$ such that for every $x \in A$, there is $m \leq m_{0}$ such that $f_{i_{m}(x)} \circ \cdots \circ f_{i_{1}(x)}(x) \in U$. Since Ls $F^{n}\left(\left\{x_{0}\right\}\right) \subset A$ then $x_{n}=f_{\omega}^{n}\left(x_{0}\right) \in V$ for all $n$ large enough. Then $E_{n}=\left\{\omega \in \Omega: \omega_{n+m_{0}}=i_{m_{0}}\left(x_{n}\right), \ldots, \omega_{n+1}=i_{1}\left(x_{n}\right)\right\}$ is obviously contained in $E$, and since the random variable $x_{n}$ depends only on $\omega_{1}, \ldots, \omega_{n}$, we obtain from (1) that

$$
\mathbb{P}\left(E \mid \omega_{1}, \ldots, \omega_{n}\right) \geq \mathbb{P}\left(E_{n} \mid \omega_{1}, \ldots, \omega_{n}\right) \geq p^{m_{0}} \stackrel{\text { def }}{=} \delta_{0} .
$$

In particular, for every set $C$ in the $\sigma$-algebra generated by $\omega_{1}, \ldots, \omega_{n}$, we have the inequality $\mathbb{P}(E \cap C) \geq \delta_{0} \mathbb{P}(C)$, and since $n$ is arbitrary, this inequality actually holds for any Borel set $C$ of $\Omega$. Choosing $C=\Omega \backslash E$, we deduce that $\mathbb{P}(\Omega \backslash E)=0$ concluding that $E$ has full probability.

Finally, we will prove that with probability $1, O_{\omega}^{+}\left(x_{0}\right)$ is dense in $A$. First notice that any quasi-attractor is a separable set. Hence, let us choose $\left(z_{i}\right)_{i \in \mathbb{N}}$ a sequence dense in $A$ and for each $i,\left(U_{i, j}\right)_{j \in \mathbb{N}}$ a basis of neighborhood of $z_{i}$. Let

$$
\Omega\left(x_{0}\right)=\bigcap_{i \in \mathbb{N}} \bigcap_{j \in \mathbb{N}} E\left(x_{0}, U_{i, j}\right) .
$$

From the above, $\Omega\left(x_{0}\right)$ has full probability. Given any open set $U$ of $X$ so that $U \cap A \neq \emptyset$. Then we find $U_{i, j}$ so that $U_{i, j} \subset U$. Then, for every $\omega \in \Omega\left(x_{0}\right)$, the set $O_{\omega}^{+}\left(x_{0}\right)$ intersects $U_{i, j}$ and in particular $U$. Thus $O_{\omega}^{+}\left(x_{0}\right)$ is dense in $A$.

\section{On Leśniak deterministic chaos game for quasi-attractor of non-expansive IFS:}

Theorem. Every quasi-attractor of a non-expansive IFS on a metric space is renderable by the deterministic chaos game.

Proof. We will use Lemma 3.14. To accomplish this, let $I$ be an open set so that $A \cap I \neq \emptyset$. We can suppose that $I=B_{2 \varepsilon}\left(y_{0}\right)$ is an open ball of radius $2 \varepsilon>0$ and centered at $y_{0} \in A$. By the compactness of $A$, we can find $y_{1}, \ldots, y_{m} \in A$ such that $A \subset B_{\varepsilon}\left(y_{0}\right) \cup B_{\varepsilon}\left(y_{1}\right) \cup \cdots \cup B_{\varepsilon}\left(y_{m}\right) \stackrel{\text { def }}{=} Z$. Being the action of $\Gamma$ on $A$ minimal, we find $h_{1} \in \Gamma$ such that $h_{1}\left(y_{1}\right) \in B_{\varepsilon}\left(y_{0}\right)$. Recursively, constructed $h_{i-1}$ we find $h_{i} \in \Gamma$ such that $h_{i} \circ \cdots \circ h_{1}\left(y_{i}\right) \in B_{\varepsilon}\left(y_{0}\right)$. On the other hand, for each $z \in Z$, there is $i \in\{0, \ldots, m\}$ such that $d\left(z, y_{i}\right) \leq \varepsilon$. Since the IFS is non-expansive,

$$
d\left(h_{i} \circ \cdots \circ h_{1}(z), h_{i} \circ \cdots \circ h_{1}\left(y_{i}\right)\right) \leq d\left(z, y_{i}\right) \leq \varepsilon .
$$

Since $d\left(h_{i} \circ \cdots \circ h_{1}\left(y_{i}\right), y_{0}\right) \leq \varepsilon$ it follows that $d\left(h_{i} \circ \cdots \circ h_{1}(z), y_{0}\right) \leq 2 \varepsilon$. That is, $h_{i} \circ \cdots \circ h_{1}(z) \in I$ for some $i \in\{0, \ldots, m\}$ where we recall that $h_{i} \circ \cdots \circ h_{1}$ for $i=0$ denotes the identity map. Hence writing

$$
f_{i_{s}} \circ \cdots \circ f_{i_{1}}=h_{m} \circ \cdots \circ h_{1} \quad \text { where } f_{i_{j}} \in \mathscr{F}
$$

we have obtained that there is $t \in\{0, \ldots, s\}$ so that $f_{i_{t}} \circ \cdots \circ f_{i_{1}}(z) \in I$. Thus, by Lemma 3.1.1, $A$ is renderable by the deterministic chaos game. 


\section{REFERENCES}

[1] V. A. Antonov, Modeling Cyclic Evolution Processes: Synchronization by Means of Random Signal, Universitet Vestnik Matematika Mekhanika Astronomiia, (1984), 67-76.

[2] T. Banakh, W. Kubiś, N. Novosad, M. Nowak and F. Strobin Contractive function systems, their attractors and metrization, Topological Methods in Nonlinear Analysis, Volume 46, Number 2 (2015), 1029-1066.

[3] M.F. Barnsley, Fractal Everywhere, Academic Press Professional, Inc, 1988.

[4] M. Barnsley and K. Igudesman, Topological Contractive Systems, Lobachevskii Journal of Mathematics, 32 (3), (2011), 220-223.

[5] M. F. BARNSLEY AND K. LeŚNIAK, The chaos game on a general iterated function system from a topological point of view, International Journal of Bifurcation and Chaos, 24 (2014).

[6] _ On the Continuity of the Hutchinson Operator, Symmetry, 7 (4), (2015), 1831-1840.

[7] M. F. BARnsLEY, K. LeŚniAK And M. Rypka, Chaos game for IFSs on topological spaces, Journal of Mathematical Analysis and Applications, Vol. 435, Issue 2, (2016), 1458-1466.

[8] M. F. Barnsley, K. Leśniak and C. Wilson, Some Recent Progress Concerning Topology of Fractals, Recent Progress in General Topology III, (2014), 69-92.

[9] M. F. Barnsley And A. Vince, The chaos game on a general iterated function system, Ergod. Th. \& Dynam. Sys., 31 (2011), 1073-1079.

[10] __ Developments in fractal geometry, Bull. Aust. Math. Soc., 3 (2013), 299-348.

[11] P. G. Barrientos, A. Fakhari and A. Sarizadeh, Weakly hyperbolic and fiberwise orbits, extended abstract to IMC44, 2013.

[12] _ Density of fiberwise orbits in minimal iterated function systems on the circle, Discrete and Continuous Dynam. Systems, 34, no. 9 (2014), 3341-3352.

[13] P. G. Barrientos, A. Fakhari, D. Malicet and A. Sarizadeh, Expanding actions: minimality and ergodicity, Stochastics and Dynamics, 17 (2017), 1750031-1750051.

[14] P. G. Barrientos And A. Raibekas, Dynamics of iterated function systems on the circle close to rotations, Ergod. Th. \& Dynam. Sys., 35 no. 5 (2015), 1345-1368.

[15] G. Bee, Topologies on closed and closed convex sets, Mathematics and its Applications, 268, 1993.

[16] R. Bowen, A horseshoe with positive measure, Inventiones Mathematicae, 29 no. 3 (1975), 203-204.

[17] L. Breiman The Strong Law of Large Numbers for a Class of Markov Chains, Ann. Math. Statist. 31, no. 3 (1960), 801-803.

[18] G. A. Edgar, Integral, Probability, and Fractal Measures, Springer-Verlag, 1998.

[19] A. Furman, Random walks on groups and random transformations, Handbook of dynamical systems, Vol. 1A, pp. 931-1014, North-Holland, 2002

[20] E. GHys, Groups acting on the circle, Enseign. Math. (2),47 (2001), 329-407.

[21] T. Golenishcheva-Kutuzova, A. S. Gorodetski, V. Kleptsyn and D. Volk, Translation numbers define generators of $F_{k}^{+} \rightarrow$ Homeo $_{+}\left(\mathbb{S}^{1}\right)$, Moscow Mathematical Journal, 14 no. 2 (2014), 291-308.

[22] B. Kieninger, Iterated Function Systems on Compact Hausdorff Spaces, Ph.D. Thesis, Augsburg University, Berichte aus der Mathematik, Shaker-Verlag, Aachen 2002.

[23] V. A. Kleptsyn And M. B. NAlski, Contraction of orbits in random dynamical systems on the circle, Functional Analysis and Its Applications, 38 (2004), 267-282.

[24] A. Lasota and J. Myjak Semifractals, Bull. Polish Acad. Sci. Math. 44 (1996), 5-21.

[25] _ Fractals, semifractals and Markov operators, International Journal of Bifurcation and Chaos 9 no. 2 (1999), 307-325.

[26] A. Lasota, J. Myjak and T. Szarek, semifractals and Markov operators, Progress in Probability, 57 (2004), 3-22.

[27] K. LEŚNIAK, Random iteration for nonexpansive iterated function systems: derandomised algorithm, International Journal of Applied Nonlinear Science, 1 no. 4, (2014), 360-363.

[28] _ Random iteration for infinite nonexpansive iterated function systems, Chaos, 25, (2015).

[29] D. Malicet, Random walks on Homeo $\left(S^{1}\right)$, Communications in Mathematical Physics, Volume 356, Issue 3, (2017) pp 10831116 
[30] J. R. Munkres, Topology, Pretince Hall, Incorporated, 2nd Edition 2000.

[31] S. M. Srivastava, A Course on Borel Sets, Graduate Texts in Mathematics, 1998.

[32] A. Navas, Groups of circle diffeomorphisms, University of Chicago Press, 2011.

[33] R. Miculescu, A. Minail, On a question of A. Kameyama concerning self-similar metrics J. Math. Anal. Appl. 422 (2015), 265-271.

Pablo G. Barrientos, Instituto de Matemática e Estatística, Universidade Federal Fluminense, Rua Mário Santos Braga s/n - Campus Valonguinhos, Niterói, Brazil

E-mail address: pgbarrientos@id.uff.br

Fatemeh H. Ghane, Department of Mathematics, Ferdowsi University of Mashhad, Mashhad, Iran

E-mail address: ghane@math.um.ac.ir

Dominique Malicet, Instituto de Matemática e Estatística, Universidade do Estado do Rio de Janeiro, São Francisco Xavier, 524 - Pavilhão Reitor João Lyra Filho, Rio de Janeiro, Brazil

E-mail address: malicet@mat.puc-rio.br

Aliasghar Sarizadeh, Department of Mathematics, Ilam University, Ilam, Iran

E-mail address: a.sarizadeh@mail.ilam.ac.ir 